\title{
Strong $(1,1,2)$-Kernels in the Corona of Graphs and Some Realization Problems
}

\author{
Urszula Bednarz ${ }^{1}$ (D)
}

Received: 25 March 2019/ Accepted: 21 January 2020 / Published online: 10 February 2020

(C) The Author(s) 2020

\begin{abstract}
In this paper, we give the necessary and sufficient conditions for the existence of strong $(1,1,2)$-kernels in the corona of graphs. Moreover, we consider lower and upper strong $(1,1,2)$-kernel numbers and we prove that the difference between these parameters can be arbitrarily large.
\end{abstract}

Keywords Domination · Independence $\cdot$ Kernel · Corona

Mathematics Subject Classification 05C20 05 C69

\section{Introduction and Preliminary Results}

In general, we use the standard terminology and notation of graph theory, see Diestel (2005). Let $G$ be an undirected, connected, simple graph with the vertex set $V(G)$ and the edge set $E(G)$. Then, the order of $G$ is its number of vertices and the size of $G$ is its number of edges. For a vertex $x \in V(G)$, let $\operatorname{deg}_{G}(x)$ denote its degree. By $P_{n}$ for $n \geq 2$, and $C_{n}$ for $n \geq 3$, we mean a path and a cycle of order $n$, respectively. Let $T_{n}, n \geq 2$, be a tree of order $n$. In any tree, a vertex of degree 1 is a leaf and $L(G)=\{x \in$ $\left.V(G): \operatorname{deg}_{G}(x)=1\right\}$. A vertex adjacent to at least one leaf is a support vertex.

By $d_{G}(x, y)$, we denote the distance between vertices $x$ and $y$ in $G$ being the length of a shortest path between $x$ and $y$. Let $J \subset V(G)$. Then, $d_{G}(x, J)=\min _{y \in J} d_{G}(x, y)$. The diameter of $G$ is the number $\operatorname{diam}(G)=\max _{x, y \in V(G)}$ $d_{G}(x, y)$. A tree containing exactly two support vertices is called a 2-palm. If a 2-palm has the diameter 3, then we call it a double star. A double star $P\left(n_{1}, n-n_{1}-2\right)$ of order $n \geq 5$ and diameter 3 is a tree with the support vertex

Urszula Bednarz

ubednarz@prz.edu.pl

1 Department of Discrete Mathematics, Faculty of Mathematics and Applied Physics, Rzeszow University of Technology, al. Powstańców Warszawy 12, 35-359 Rzeszow, Poland $x$ adjacent to $n_{1}$ leaves and the support vertex $y$ adjacent to $n-n_{1}-2$ leaves. Clearly, $x$ and $y$ are adjacent.

For any vertex $v \in V(G)$, the open neighborhood of $v$ we define as $N_{G}(v)=\{u \in V(G): u v \in E(G)\}$.

Let $G$ be a graph with $V(G)=\left\{x_{1}, \ldots, x_{n}\right\}, n \geq 2$ and $\mathcal{H}=\left(H_{i}\right)_{i \in \mathcal{I}=\{1, \ldots, n\}}$ be a sequence of $n$ vertex disjoint graphs. For a nonempty graph $H_{i}, i \in \mathcal{I}$ let $V\left(H_{i}\right)=\left\{y_{1}^{i}, \ldots, y_{p_{i}}^{i}\right\}, p_{i} \geq 1$. The corona of the graph $G$ and the sequence $\mathcal{H}$ is a graph $G \circ \mathcal{H}$, such that $V(G \circ$ $\mathcal{H})=V(G) \cup \bigcup_{i \in \mathcal{I}} V\left(H_{i}\right) \quad$ and $\quad E(G \circ \mathcal{H})=E(G) \cup \bigcup_{i \in}$ $\mathcal{I} E\left(H_{i}\right) \cup \bigcup_{i \in \mathcal{I}}\left\{x_{i} y_{t}^{i}: t=1, \ldots, p_{i}\right\}$. If all graphs in the sequence $\mathcal{H}$ are isomorphic to the same graph $H$, then we obtain the corona of two graphs.

A subset $S \subseteq V(G)$ is an independent set of $G$ if no two vertices of $S$ are adjacent in $G$. A subset $D \subseteq V(G)$ is a dominating set of $G$ if each vertex not belonging to $D$ is adjacent to at least one vertex of $D$. A subset $J \subseteq V(G)$ being independent and dominating is a kernel of $G$.

The theory of kernels was initiated for digraphs by Neumann and Morgenstern in 1953 in the context of game theory, see Von Neumann and Morgenstern (1953). Since then kernels have been relevant in the graph theory for their relations with distinct problems, for example in the list colorings and perfectness. Berge was one of the pioneers in this area studying the problem of the existence of kernels in digraphs and using a kernel for solving problems in other areas of mathematics, see Berge (1973). There are many types and generalizations of kernels. Main two directions of generalizations concern $(k, l)$-kernels which generalize 
kernels in the distance sense and kernels by monochromatic paths which generalize kernels with respect to edge coloring of a graph. Many kinds of kernels are intensively studied in the literature, for details see Galeana-Sanchez and Ramirez (2007), Galeana-Sanchez and Sanchez-Lopez (2011), Kucharska (2005), Szumny et al. (2008) and Włoch (2008). Kernels by monochromatic paths are studied only in digraphs because in undirected graphs the existing problem is trivial. The problem of the existence of $(k, l)$-kernels in undirected graphs is interesting only for special values of $k, l$.

In an undirected graph, every maximal independent set is its kernel, since every maximal independent set is also a dominating set. The problem is more complicated if we add restrictions related to the domination or the independence. Then, new types of kernels are defined and studied, among others efficient dominating sets (Bange et al. 1988), secondary domination (Hedetniemi et al. 2008), restrained independent dominating sets (Domke et al. 1999).

In Włoch (2012), Włoch introduced the concept of a 2dominating kernel in graphs (shortly (2-d)-kernel). A subset $J \subset V(G)$ is a 2-dominating kernel of $G$ if $J$ is independent and 2-dominating, i.e., $J$ is independent and each vertex from $V(G) \backslash J$ has at least two neighbors in $J$. Some properties of (2-d)-kernels were studied in Bednarz et al. (2015), Bednarz and Włoch (2016), Bednarz and Włoch (2017). In Nagy (2016), Nagy introduced and studied a more general concept, namely a $k$-dominating kernel for $k \geq 2$.

In Hedetniemi et al. (2008), the authors introduced the definition of a $(1,2)$-dominating set. A set $S \subseteq V(G)$ is called a (1,2)-dominating set of $G$ if for every vertex $v \in$ $V(G) \backslash S$ there are two distinct vertices $u, w \in S$, such that $u$ is adjacent to $v$, and $w$ is within distance 2 of $v$ (i.e., $\left.d_{G}(v, w) \leq 2\right)$. This kind of domination is called also as secondary domination. In this terminology, a 2-dominating set is the same as a $(1,1)$-dominating set. In particular if for a vertex $v \in V(G) \backslash S$ there exists a vertex $u \in S$, such that $d_{G}(v, w)=2$ we say that $v$ is 2-distance dominated by $S$. In Hedetniemi et al. (2008), the authors considered also a $(1,2)$-kernel called as an independent $(1,2)$-dominating set and they obtained some properties of this set.

In [5], the authors introduced and studied some new types of kernels based on (2-d)-kernels and $(1,2)$-kernels as a natural consequence.

A subset $J \subseteq V(G)$ is a $(1,1,2)$-kernel of $G$ if $J$ is independent and $(1,1,2)$-dominating i.e., for each vertex $x \notin J$ there are three distinct vertices $y_{1}, y_{2}, y_{3} \in J$ such that $x y_{i} \in E(G)$, for $i=1,2$ and $d_{G}\left(x, y_{3}\right) \leq 2$. Clearly, every $(1,1,2)$-kernel is the classical kernel, the $(1,2)$-kernel and the 2-dominating kernel.

Let $J$ be a $(1,1,2)$-kernel. If for every vertex $x \notin J$ there exists $y \in J$, such that $d_{G}(x, y)=2$, then $J$ is a strong $(1,1,2)$-kernel. In other words $J$ is a strong $(1,1,2)$-kernel if $J$ is independent and strong $(1,1,2)$-dominating. Moreover, for a graph $G$ being totally disconnected the set $V(G)$ is a $(1,1,2)$-kernel and a strong $(1,1,2)$-kernel.

Below we give some necessary conditions for the existence of the $(1,1,2)$-kernels, see [5].

(i) If $J$ is a $(1,1,2)$-kernel (strong $(1,1,2)$-kernel) of $G$, then $|J| \geq 3$.

(ii) If $J$ is a $(1,1,2)$-kernel (strong $(1,1,2)$-kernel) of $G$, then $L(G) \subset J$.

(iii) If $J$ is a $(1,1,2)$-kernel (strong $(1,1,2)$-kernel) of $G$, then for every $x \notin J$, it holds $\operatorname{deg}_{G}(x) \geq 3$.

(iv) $\quad P_{n}$ and $C_{n}$ does not have a $(1,1,2)$-kernel (strong $(1,1,2)$-kernel).

There was given a complete characterization of trees with a strong $(1,1,2)-k e r n e l$ in the paper [5]. Moreover, this type of kernel was studied in generalized Petersen graph and the join of graphs.

Theorem 1 A subset $J \subseteq V(G)$ is a strong $(1,1,2)$-kernel if and only if $J$ is a maximal independent set and $J$ is a minimal strong $(1,1,2)$-dominating set.

Proof Assume that a subset $J \subseteq V(G)$ is a maximal independent set and $J$ is a minimal strong $(1,1,2)$-dominating set. Then, by the definition, $J$ is a strong $(1,1,2)$ kernel.

Conversely, let $J \subseteq V(G)$ be a strong $(1,1,2)$-kernel of a graph $G$. From the definition of a strong $(1,1,2)$-kernel, it is clear that $J$ is an independent set and $J$ is a strong $(1,1,2)$-dominating set. We prove that $J$ is a maximal independent set. Suppose on the contrary that $J$ is not a maximal independent set. Then, there exists a vertex $x \notin J$, such that $J \cup\{x\}$ is an independent set. Therefore, $N(x) \cap$ $J=\emptyset$ which is a contradiction to the fact that $x$ is dominated by $J$. Hence, $J$ is the maximal independent set.

Now suppose on the contrary that $J$ is not a minimal strong $(1,1,2)$-dominating set. Then, there exists a vertex $u \in J$, such that $J \backslash\{u\}$ is still a strong $(1,1,2)$-dominating set. It implies that $u$ is dominated by $J \backslash\{u\}$; hence, there exists a vertex $y \in J \backslash\{u\}$ adjacent to $u$, which is a contradiction to the fact that $J$ is an independent set. Therefore, $J$ is a minimal strong $(1,1,2)$-dominating set.

Thus ends the proof.

Let $I_{(1,1,2)}(G)$ be the largest cardinality of a strong $(1,1,2)$-kernel of a graph $G$, and let $i_{(1,1,2)}(G)$ be the smallest cardinality of this kernel.

Theorem 2 (Realization Theorem) For an arbitrary integer $n \geq 1$, there exists a graph $G$, such that 


$$
I_{(1,1,2)}(G)-i_{(1,1,2)}(G)=n
$$

Proof Let $n \geq 1$ be an integer. To prove this theorem, it suffices to construct a graph $G$, such that equality (1) holds. To construct it, let us consider a double star $P(2, n+6)$ of order $n+10$ with support vertices $x, y$. We define a graph $G$ as follows $V(G)=V(P(2, n+6))$ and $E(G)=E(P(2, n+6)) \cup E_{0}$, where $E_{0}$ is the set of $2(n+$ $3)$ edges added to the double star $P(2, n+6)$. To describe the set $E_{0}$, let us consider the set $V_{0}$ of $n+6$ leaves adjacent to the support vertex $x$ in the double star $P(2, n+6)$. On the set $V_{0}$, we define a 2-coloring function by distinguishing three red vertices and $n+3$ blue. Let $B$ be the set of all blue vertices, and let $R$ be the set of red vertices. Then, $V_{0}=B \cup R$ and clearly $B \cap R=\emptyset$. The set $E_{0}$ contains edges joining blue and red vertices, such that every blue vertex is joined with exactly two red vertices, assuming that none of the red vertices is adjacent to all $n+3$ blue vertices. It is clear that each red vertex has at least two blue neighbors. By $\left\{z_{1}, z_{2}\right\}$, we denote the set of two leaves adjacent to the support vertex $y$. We show that the set $J_{1}=\left\{z_{1}, z_{2}\right\} \cup R$ is the smallest strong $(1,1,2)$ kernel of $G$ and the set $J_{2}=\left\{z_{1}, z_{2}\right\} \cup B$ is the largest strong $(1,1,2)$-kernel of $G$. The independence of $J_{1}$ and $J_{2}$ is obvious. Firstly, we shall show that $J_{1}$ and $J_{2}$ are strong $(1,1,2)$-dominating sets. Clearly, $x, y \notin J_{1}$ and $x, y \notin J_{2}$. Moreover, the vertex $y$ is 2-dominated by vertices $z_{1}, z_{2}$ and $d_{G}(y, B)=2$ and $d_{G}(y, R)=2$. Hence, the support vertex $y$ is strong $(1,1,2)$-dominated by $J_{1}$ and $J_{2}$. Analogously, we can show that the support vertex $x$ and vertices from the set $B$ are strong $(1,1,2)$-dominated by $J_{1}$ and $J_{2}$. From the above, $\left|J_{1}\right|=|R|+|L(x)|=5$ and $J_{1}$ is the smallest strong $(1,1,2)$-kernel of $G$. Moreover, $\left|J_{2}\right|=|B|+|L(x)|=n+$ 5 and $J_{2}$ is the largest strong $(1,1,2)$-kernel of $G$. Consequently, $I_{(1,1,2)}(G)-i_{(1,1,2)}(G)=n$. Thus, the theorem is proved.

A dominating set $S \subseteq V(G)$ is a restrained dominating set if every vertex $v \in V(G) \backslash S$ is adjacent to a vertex in $S$ and another vertex in $V(G) \backslash S$. A restrained dominating set which is also independent is called a restrained kernel. The concept of restrained domination in graphs was introduced by Telle and Proskurowski (1997), albeit indirectly, as a vertex partition problem. The restrained domination and total restrained domination were studied in Domke et al. (1999) and Chen et al. (2011).

Theorem 3 If $J \subseteq V(G)$ is a strong $(1,1,2)$-kernel, then $J$ is a restrained kernel.

Proof Suppose that $J$ is a strong $(1,1,2)$-kernel. Then, $J$ is an independent set and $J$ is a strong $(1,1,2)$-dominating set. Therefore for each vertex $x \notin J$, there exists a vertex $y \in J$, such that $d_{G}(x, y)=2$. Consequently, there exists a vertex $z \notin J$ adjacent to $x$. And also from the fact that $J$ is a (1,1)-dominating set there exists a vertex $u \in J$ adjacent to $x$. Hence, a vertex $x$ is adjacent to $z \notin J$ and to $y \in J$. By the assumption that $J$ is an independent set, it follows that $J$ is the restrained kernel.

\section{Strong $(1,1,2)$-Kernels in the Corona of Graphs}

In this section, we study the existence of strong $(1,1,2)$ kernels in the corona $G \circ \mathcal{H}$. We give the necessary and sufficient conditions for the existence of strong $(1,1,2)$ kernels in this graph product. We consider this problem depending on whether all graphs $H_{i}$ for $i=1, \ldots, n$ are nonempty or at least one of them has an order at most 1. The existence of distinct types of kernels in $G \circ \mathcal{H}$ has been investigated, see for example in Szumny et al. (2008) and Włoch (2008).

Theorem 4 Let $G$ be a connected graph of order $n \geq 2$ and $\mathcal{H}$ be a sequence of $n$ vertex disjoint nonempty graphs $H_{1}, \ldots, H_{n}$ of order $p_{i} \geq 2$. The graph $G \circ \mathcal{H}$ has a strong $(1,1,2)-k e r n e l$ if and only if $H_{i}$ has a strong $(1,1,2)$ kernel, for $i=1, \ldots, n$.

Proof Let $G$ be a graph of order $n \geq 2$ and suppose that $H_{1}, \ldots, H_{n}$ are vertex disjoint nonempty graphs. Suppose that $H_{i}$ has a strong $(1,1,2)$-kernel $J_{i}$, for $i=1, \ldots, n$. We shall show that $J=\bigcup_{i=1}^{n} J_{i}$ is a strong $(1,1,2)$-kernel of $G \circ \mathcal{H}$. The independence of $J$ is obvious, then it suffices to show that $J$ is a strong $(1,1,2)$-dominating set. Every vertex from $V\left(H_{i}\right) \backslash J$ is strong $(1,1,2)$-dominated by $J_{i} \subset J$, for $i=1, \ldots, n$. Let us consider the vertex $x_{i} \in V(G)$. Clearly, $x_{i}$ is 2 -dominated by $J_{i}$ since $p_{i} \geq 2$ and there exists a vertex $y_{t}^{j} \in J_{i} \subset V\left(H_{j}\right)$, such that $j \neq i$ and vertices $x_{i}, x_{j}$ are adjacent in $G$. Then, by the definition of $G \circ \mathcal{H}$, it holds $d_{G \circ \mathcal{H}}\left(x_{i}, y_{t}^{j}\right)=2$. Consequently, $J$ is a strong $(1,1,2)$-kernel of $G \circ \mathcal{H}$.

Assume now that $G \circ \mathcal{H}$ has a strong $(1,1,2)$-kernel. Firstly, we show that $J \cap V(G)=\emptyset$. Suppose on the contrary that $J \cap V(G) \neq \emptyset$ and let $x_{i} \in J \cap V(G)$. Then, $V\left(H_{i}\right) \cap J=\emptyset$; hence, vertices from $V\left(H_{i}\right)$ are not 2dominated by $J$. It is a contradiction with the assumption that $J$ is a strong $(1,1,2)$-kernel. This means that $J \subset$ $\bigcup_{i=1}^{n} V\left(H_{i}\right)$ and moreover $J=\bigcup_{i=1}^{n} J_{i}$, where $J_{i}$ is a strong $(1,1,2)$-kernel of $H_{i}$. Consequently, $H_{i}$ has a strong $(1,1,2)$-kernel for all $i=1, \ldots, n$. Thus ends the proof. $\square$

From the above characterization, we obtain the value of parameters of a strong $(1,1,2)$-kernel in the corona $G \circ \mathcal{H}$. The number of all strong $(1,1,2)$-kernels in a graph $G$ we denote by $\sigma_{(1,1,2)}(G)$. 
Corollary 5 Let $G$ be a connected graph of order $n \geq 2$ and $\mathcal{H}$ be a sequence of $n$ vertex disjoint nonempty graphs $H_{1}, \ldots, H_{n}$ of order $p_{i} \geq 2$ with a strong $(1,1,2)$-kernel. Then,

$$
\begin{aligned}
I_{(1,1,2)}(G \circ \mathcal{H}) & =\sum_{i=1}^{n} I_{(1,1,2)}\left(H_{i}\right), \\
i_{(1,1,2)}(G \circ \mathcal{H}) & =\sum_{i=1}^{n} i_{(1,1,2)}\left(H_{i}\right), \\
\sigma_{(1,1,2)}(G \circ \mathcal{H}) & =\prod_{i=1}^{n} \sigma_{(1,1,2)}\left(H_{i}\right) .
\end{aligned}
$$

The problem of the existence of strong $(1,1,2)$-kernels in the corona of the graphs $G \circ \mathcal{H}$ is more complicated if for at least one of a graph $H_{i}, i=1, \ldots, n$ it holds a condition $\left|V\left(H_{i}\right)\right| \leq 1$. The next theorem gives a complete characterization of the existence of a strong $(1,1,2)$-kernel in that case.

Theorem 6 Let $G$ be a connected graph of order $n \geq 2$ and $\emptyset \neq \mathcal{H}=\left(H_{i}\right)_{i \in \mathcal{I}=\{1, \ldots, n\}}$ be a sequence of graphs, such that for at least one of a graph $H_{i}, 1 \leq i \leq n$, it holds a condition $\left|V\left(H_{i}\right)\right| \leq 1$. A subset $J^{*} \subset V(G \circ \mathcal{H})$ is a strong $(1,1,2)$ kernel if and only if $J$ is a nonempty independent set of $G$, such that $J^{*}=J \cup \bigcup_{i \in \mathcal{I}_{1}} J_{i}$, where $\mathcal{I} \supset \mathcal{I}_{1}=\left\{i: x_{i} \notin J\right\}$, $J_{i} \subseteq V\left(H_{i}\right)$ and $H_{j}=\emptyset$ for $j \in \mathcal{I} \backslash \mathcal{I}_{1}$ and for each $i \in \mathcal{I}_{1}$ there exists $x_{p} \in N_{G}\left(x_{i}\right) \backslash J$, such that $V\left(H_{p}\right) \neq \emptyset$ or $x_{i}$ is 2distance dominated by $J$. Moreover, for each $i \in \mathcal{I}_{1}$ the following conditions hold:

(i) if $\left|V\left(H_{i}\right)\right|=0$, then $x_{i}$ is 2-dominated by $J$ or

(ii) if $\left|V\left(H_{i}\right)\right|=1$, then $x_{i}$ is dominated by $J$,

and $J_{i}$ is

(a) a strong $(1,1,2)$-kernel if $N_{G}\left(x_{i}\right) \cap J=\emptyset$ or

(b) $a(2-d)$-kernel if $N_{G}\left(x_{i}\right) \cap J \neq \emptyset$.

Proof Let $G$ be a graph of order $n \geq 2$ and $\mathcal{H} \neq \emptyset$ be a sequence of graphs as in the statements of the theorem.

1. Suppose that $J$ is a nonempty independent set of a graph $G$. We shall prove that the set $J^{*}=J \cup \bigcup_{i \in \mathcal{I}_{1}} J_{i}$ is a strong $(1,1,2)$-kernel, where $\mathcal{I}_{1}=\left\{i: x_{i} \notin J\right\}$ and $J_{i} \subseteq V\left(H_{i}\right)$. Assume that for each $i \in \mathcal{I}_{1}$ there exists $x_{p} \in N_{G}\left(x_{i}\right) \backslash J$, such that $V\left(H_{p}\right) \neq \emptyset$ or $x_{i}$ is 2-distance dominated by $J$ and $V\left(H_{j}\right)=\emptyset$ for $j \in \mathcal{I} \backslash \mathcal{I}_{1}$. Suppose that the condition (i) or (ii) is valid. Assume that also the set $J_{i} \subset V\left(H_{i}\right), i \in \mathcal{I}_{1}$ satisfies the condition (a) or (b). It is obvious that $J$ and $J_{i}$ are independent sets. To prove the independence of $J^{*}$, let us consider vertices $x_{k}$ and $y_{t}^{j}$, such that $x_{k} \in J$ and $y_{t}^{j} \in J_{j}$. From the fact that $J^{*}=J \cup \bigcup_{i \in \mathcal{I}_{1}} J_{i}$, where $\mathcal{I}_{1}=\left\{i: x_{i} \notin J\right\}$, it is clear that $k \neq j$; hence, $x_{k}$ and $y_{t}^{j}$ are not adjacent. If we consider that vertices $y_{t}^{j} \in J_{j}$ and $y_{k}^{i} \in J_{i}$, then the condition of independence is obvious because the sets $H_{j}$ and $H_{i}$ are disjoint. Hence, $J^{*}$ is an independent set. Now we shall show that $J^{*}$ is a strong $(1,1,2)$-dominating set. Let us consider a vertex not belonging to $J^{*}$. Clearly, it belongs to $V\left(H_{i}\right) \backslash J_{i}, i \in \mathcal{I}_{1}$ or $V(G) \backslash J$. Without loss of generality denote these vertices by $y_{t}^{i}$ or $x_{i}$, respectively. Then, we consider the following cases:

1.1. $y_{t}^{i} \in V\left(H_{i}\right) \backslash J_{i}$ for $i \in \mathcal{I}_{1}$.

If $J_{i}$ satisfies the condition (a), then $J_{i}$ is a strong $(1,1,2)$-kernel. Hence, the vertex $y_{t}^{i}$ is strong $(1,1,2)$ dominated by $J_{i}$.

If $J_{i}$ satisfies the condition (b), then the vertex $y_{t}^{i}$ is 2dominated by $J_{i}$. Since the vertex $x_{i} \notin J$ and from the fact that $N_{G}\left(x_{i}\right) \cap J \neq \emptyset$, there exists a vertex $x_{k} \in J$, such that $d_{G}\left(y_{t}^{i}, x_{k}\right)=2$. Hence, $y_{t}^{i}$ is strong $(1,1,2)$-dominated by $J^{*}$.

1.2. $x_{i} \in V(G) \backslash J$ for $i \in \mathcal{I}_{1}$.

We distinguish the following possibilities:

If the condition (a) or (b) hold, then the vertex $x_{i}$ is 2dominated by $J_{i} \subset V\left(H_{i}\right)$. Moreover, from the assumption, there exists $x_{p} \in N_{G}\left(x_{i}\right) \backslash J$, such that $V\left(H_{p}\right) \neq \emptyset$. Hence, there is $y_{t}^{p} \in J_{p}$, such that $d_{G}\left(y_{t}^{p}, x_{i}\right)=2$. Otherwise, $x_{i}$ is 2distance dominated by $J$. Therefore, $x_{i}$ is strong $(1,1,2)$ dominated by $J^{*}$.

If the condition (i) is valid, then $\left|V\left(H_{i}\right)\right|=0$ and $x_{i}$ is 2dominated by $J$. By the assumption $x_{i}$ is 2-distance dominated by $J_{p}$ if $V\left(H_{p}\right) \neq \emptyset$ or is 2-distance dominated by $J$. If the condition (ii) holds, clearly $\left|V\left(H_{i}\right)\right|=1$, then $x_{i}$ is dominated by $J$ and is dominated by $y_{1}^{i} \in J_{i}$. Hence, $x_{i}$ is 2-dominated. Analogously as above, we can show that $x_{i}$ is 2-distance dominated. Therefore, $x_{i}$ is strong $(1,1,2)$ dominated by $J^{*}$.

2. Conversely, suppose that a set $J^{*} \subset V(G \circ \mathcal{H})$ is a strong $(1,1,2)$-kernel and the sequence of graphs $\mathcal{H}$ is as in the statements of the theorem. From the definition of $G \circ \mathcal{H}$ and by the fact that for at least one of a graph $H_{i}$, $1 \leq i \leq n$, it holds $\left|V\left(H_{i}\right)\right| \leq 1$ it is clear that $J^{*} \cap V(G) \neq \emptyset$ and $J^{*} \cap V(\mathcal{H}) \neq \emptyset$. It follows that there exists a vertex $x_{t} \in J^{*} \cap V(G)$ and then $J^{*} \cap V\left(H_{t}\right)=\emptyset$, otherwise a contradiction to the fact that $J^{*}$ is a strong $(1,1,2)$-kernel. Moreover, by the assumption, if $x_{t} \in J^{*}$, then $V\left(H_{t}\right)=\emptyset$. There also exists a vertex $y_{k}^{j} \in J^{*} \cap V\left(H_{j}\right)$ and $x_{j} \notin J^{*}$. From the above, we deduce that $J^{*}=J \cup \bigcup_{i \in \mathcal{I}_{1}} J_{i}$, where $J \subset V(G), J_{i} \subseteq V\left(H_{i}\right)$ and $\mathcal{I}_{1}=\left\{i: x_{i} \notin J\right\}, V\left(H_{j}\right)=\emptyset$ for $j \in \mathcal{I} \backslash \mathcal{I}_{1}$. Because for at least one of the graph $H_{i}$ there is $\left|V\left(H_{i}\right)\right| \leq 1$, it is clear that $J \subset V(G)$ is nonempty. It is also obvious that $J$ and $J_{i}$ for $i=1, \ldots, n$, are independent sets. Let us consider an arbitrary vertex from $V(G \circ \mathcal{H}) \backslash J^{*}$. Then, by the definition of $J^{*}$, it does not belong to $J$ and to $J_{i}, i \in \mathcal{I}_{1}$. Since $J^{*}$ is a strong $(1,1,2)$ - 


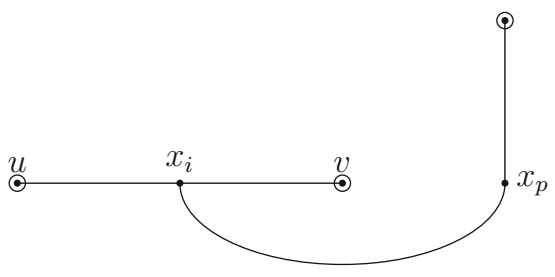

or

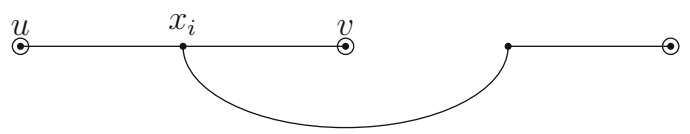

Fig. 1 The case when $\left|V\left(H_{i}\right)\right|=0$

kernel, then there exist three vertices belonging to $J^{*}$, such that two of them are adjacent to considered vertex and there is a vertex in $J^{*}$ being at the distance 2 from a given vertex. Let us consider an arbitrary vertex $x \notin J^{*}$ and firstly assume that $x \in V(G) \backslash J$, we denote this vertex as $x_{i}$, $i \in \mathcal{I}_{1}$. From the fact that $x_{i}$ is 2-distance dominated by $J^{*}$, there exists a vertex $x_{p} \in N_{G}\left(x_{i}\right) \backslash J$, such that $V\left(H_{p}\right) \neq \emptyset$ and then $d_{G \circ \mathcal{H}}\left(x_{i}, y_{t}^{p}\right)=2$ for $y_{t}^{p} \in J_{p} \subset V\left(H_{p}\right)$ or there exists a vertex $x_{t} \in J$, such that $d_{G}\left(x_{i}, x_{t}\right)=2$, i.e., $x_{i}$ is 2-distance dominated by $J$.

Since a vertex $x_{i}$ is 2-dominated by $J^{*}$ there must exist two vertices $u, v \in N_{G}\left(x_{i}\right)$, such that $u, v \in J^{*}$. We consider the following cases:

2.1. $\left|V\left(H_{i}\right)\right|=0$. Then, $u, v \in J \subset V(G)$. Hence, $x_{i}$ is 2-dominated by $J$ and the condition (i) holds. This situation is presented on Fig. 1 taking account above considerations related to 2-distance domination.

2.2. $\left|V\left(H_{i}\right)\right|=1$. Clearly, $V\left(H_{i}\right)=\{u\}$ and then a vertex $u \in J_{i}$ dominates the vertex $x_{i}$ and it is obvious that $u$ is adjacent to $x_{i}$. There also exists a vertex $v \in J \subset V(G)$ which dominates the vertex $x_{i}$. Hence, $x_{i}$ is dominated by $J$ and the condition (ii) holds. See Fig. 2.

2.3. $\left|V\left(H_{i}\right)\right| \geq 2$. Then, we consider the following possibilities:

2.3.1. if $N_{G}\left(x_{i}\right) \cap J=\emptyset$, then $u, v \in J_{i} \subset V\left(H_{i}\right)$ and $J_{i}$ must be a strong $(1,1,2)$-kernel. Otherwise, each vertex $y_{p_{i}}^{i}, p_{i}>1$, such that $y_{p_{i}}^{i} \in V\left(H_{i}\right) \backslash J_{i}$ is not strong $(1,1,2)$ dominated by $J^{*}$. Hence, the condition (a) holds. See Fig. 3.

2.3.2. if $N_{G}\left(x_{i}\right) \cap J \neq \emptyset$, then $u \in J$ and it is necessary that a graph $H_{i}$ has a (2-d)-kernel $J_{i}$. Therefore, a vertex $v \in J_{i}$ and each vertex $y_{p_{i}}^{i}, p_{i}>1$, such that $y_{p_{i}}^{i} \in V\left(H_{i}\right) \backslash J_{i}$ is 2-dominated by $J_{i}$ and $d_{G \circ \mathcal{H}}\left(u, y_{p_{i}}^{i}\right)=2$, hence $y_{p_{i}}^{i}$ is strong $(1,1,2)$-dominated by $J^{*}$. See Fig. 4.

Assuming that the vertex $x \notin J^{*}$ belongs to $V\left(H_{i}\right) \backslash J_{i}$ we prove analogously as in cases 2.3.1. and 2.3.2 for the vertex $y_{p_{i}}^{i}$.

Thus, the theorem is proved.

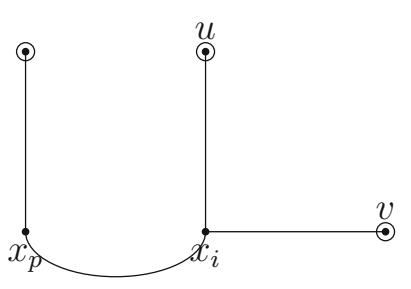

or

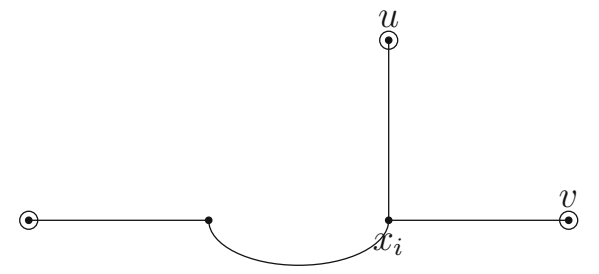

Fig. 2 The case when $\left|V\left(H_{i}\right)\right|=1$

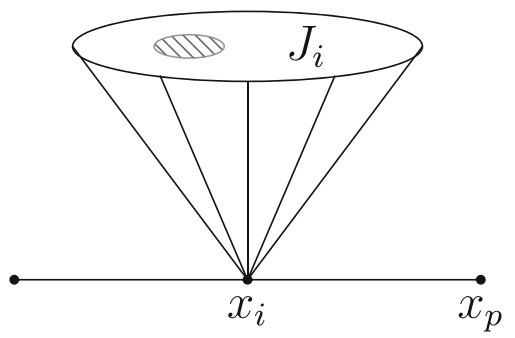

Fig. $3 J_{i}$ is a strong $(1,1,2)$-kernel

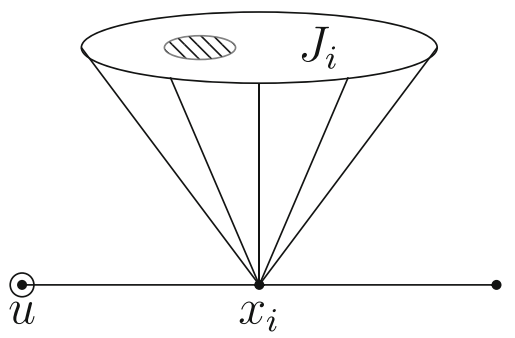

Fig. $4 J_{i}$ is a (2-d)-kernel

Open Access This article is licensed under a Creative Commons Attribution 4.0 International License, which permits use, sharing, adaptation, distribution and reproduction in any medium or format, as long as you give appropriate credit to the original author(s) and the source, provide a link to the Creative Commons licence, and indicate if changes were made. The images or other third party material in this article are included in the article's Creative Commons licence, unless indicated otherwise in a credit line to the material. If material is not included in the article's Creative Commons licence and your intended use is not permitted by statutory regulation or exceeds the permitted use, you will need to obtain permission directly from the copyright holder. To view a copy of this licence, visit http://creativecommons. org/licenses/by/4.0/. 


\section{References}

Bange DW, Barkauskas AE, Slater PJ (1988) Efficient dominating sets in graphs. Applications of discrete mathematics. SIAM, Philadelphia, pp 189-199

Bednarz P, Włoch I (2016) On (2-d)-kernels in the cartesian product of graphs. Ann UMCS Sect A 70(2):1-8

Bednarz P, Włoch I (2017) An algorithm determining (2-d)-kernels in trees. Util Math 102:215-222

Bednarz P, Hernandez-Cruz C, Włoch I (2015) On the existence and number of (2-d)-kernels in graphs. Ars Comb 121:341-351

Bednarz U, Włoch I On strong $(1,1,2)$-kernels in graphs. Ars Comb (in print)

Berge C (1973) Graphs and hypergraphs. North-Holland, Amsterdam

Chen X, Liu J, Meng J (2011) Total restrained domination in graphs. Comput Math Appl 62:2892-2898

Diestel R (2005) Graph theory. Springer, Berlin

Domke GS, Hattingh JH, Hedetniemi ST, Laskar RC, Markus LR (1999) Restrained domination in graphs. Discrete Math 203:61-69

Galeana-Sanchez H, Ramirez LA (2007) Monochromatic kernelperfectness of special classes of digraphs. Discuss Math Graph Theory 27(3):389-400
Galeana-Sanchez H, Sanchez-Lopez R (2011) $H$-kernels in the $D$ join. Ars Comb 99:353-377

Hedetniemi SM, Hedetniemi ST, Knisely J, Rall DF (2008) Secondary domination in graphs. AKCE J Graphs Comb. $5(2): 103-115$

Kucharska M (2005) On $(k, l)$-kernel perfectness of special classes of digraphs. Discuss Math Graph Theory 25:103-119

Nagy Z (2016) On the number of $k$-dominating independent sets. J Graph Theory 84(4):566-580

Szumny W, Włoch A, Włoch I (2008) On the existence and on the number of $(k, l)$-kernels in the lexicographic product of graphs. Discrete Math 308:4616-4624

Telle JA, Proskurowski A (1997) Algorithms for vertex partitioning problems on partial $k$-trees. SIAM J Discrete Math 10:529-550

Von Neumann J, Morgenstern O (1953) Theory of games and economic behavior. Princeton University Press, Princeton

Włoch I (2008) On kernels by monochromatic paths in the corona of digraphs. Cent Eur J Math 6(4):537-542

Włoch A (2012) On 2-dominating kernels in graphs. Aust J Comb 53:273-284 